\title{
Activite Antifongique Des Extraits Aqueux De Calendula Officinalis L, Urginea Maritima (L.) Baker Et Chenopodium Ambrosioides $\mathrm{L}$.
}

\section{Daoudi Amine, Bammou Mohamed,}

Equipe de Microbiologie du Sol et de l'Environnement, Département de Biologie, Faculté des Sciences, Université Moulay Ismail, Meknès, Maroc

\section{Pr. Haloui Zoubida,}

Equipe de Valorisation des Plantes Médicinales et Aromatiques de la region Meknès-Tafilalet, Département de Biologie, Faculté des Sciences, Université Moulay Ismail, Meknès, Maroc

\section{Pr. Ibijbijen Jamal, \\ Pr. Nassiri Laila,}

Equipe de Microbiologie du Sol et de l'Environnement, Département de Biologie, Faculté des Sciences, Université Moulay Ismail, Meknès, Maroc

Abstract

Fungi are the cause of many human, animal and plant diseases. In the context of valorization of aromatic and medicinal plants of the Central Middle Atlas of Morocco, three plants were selected, based on the results of an ethnobotanical survey in this region, to evaluate their antifungal activity against five Fungi, namely Fusarium oxysporum sp. albedinis, Alternaria sp, Penicillium expansum, Aspergillus brasiliensis and Rhizopus stolonifer.

A quantification of the total polyphenols was mesured out for the aqueous extracts of the studied plants Urginea maritima (L.) Baker of the Liliaceae family, Chenopodium ambrosoides L. of the Chenopodiaceae Family and Calendula officinalis $L$ of the Asteraceae family, to choose the form in which the tests will be performed. According to the results, Aspergillus brasiliensis was found sensitive to the macerate of Urginea maritima (L.) Baker at a concentration of $10.71 \mathrm{mg} / \mathrm{ml}$. The percent of inhibition (PI) of mycelial growth was $43.33 \%$. At the same concentration, Fusarium oxysporum showed a slight sensitivity with PI $=19.25 \%$. Furthermore the Chenopodium ambrosoides L decoctate was active against Aspergillus brasiliensis, Alternaria sp and Fusarium oxysporum with PI respectively equal to $41.11 \%$, 
$40.37 \%$ and $27.77 \%$. However, the infusion of Calendula officinalis L showed no effect on the strains tested.

Keywords: Antifungal activity, aqueous extracts, aromatic and medicinal plants, Central Middle Atlas, Morocco.

\section{Resume}

Les champignons sont à l'origine de nombreuses maladies humaines, animales ou végétales. Dans le cadre de la valorisation des plantes aromatiques et médicinales de la région du Moyen Atlas central du Maroc, des plantes ont été choisies, en fonction des résultats d'une enquête ethnobotanique réalisée dans cette région, afin d'évaluer leur activité antifongique contre cinq champignons, à savoir Fusarium oxysporum sp. albedinis, Alternaria sp, Penicillium expansum, Aspergillus brasiliensis et Rhizopus stolonifer. Une quantification des polyphénols totaux a été effectuée sur des extraits aqueux des plantes étudiées à savoir Urginea maritima (L.) Baker famille des Liliacées, Chenopodium ambrosoides L. famille des Chenopodiacées et Calendula officinalis L de la famille des Asteracées, pour choisir la forme sous laquelle les tests seront réalisés. D’après les résultats obtenus, Aspergillus brasiliensis s'est révélé la souche la plus sensible vis-à-vis du macérât de Urginea maritima (L.) Baker, à une concentration de $10,71 \mathrm{mg} / \mathrm{ml}$ le pourcentage d'inhibition (PI) de la croissance mycélienne égale à $43,33 \%$, à la même concentration Fusarium oxysporum a montré une légère sensibilité avec PI $=19,25 \%$.Alors que, le décocté de Chenopodium ambrosoides L s'est monté active contre Aspergillus brasiliensis, Alternaria sp et Fusarium oxysporum avec des PI égaux à $41,11 \%, 40,37 \%$ et $27,77 \%$ respectivement. L'infusé de Calendula officinalis L n'a montré aucun pouvoir vis-à-vis des souches testées.

Mots-cles: Activité antifongique, extraits aqueux, plantes aromatiques et médicinales, Moyen Atlas central, Maroc.

\section{Introduction}

Les champignons représentent un danger pour l'homme, l'animal et le végétal, leur danger résulte des dégâts qui peuvent toucher les produits agricoles, les denrées alimentaires et autres (Badji et al., 2005).

Par conséquent, le recourt à des sources végétale devraient être beaucoup plus avantageux que l'utilisation des pesticides, car elles se décomposent facilement, ne sont pas des polluants environnementaux et ne possèdent pas de propriétés résiduelles ou phytotoxiques (Sauphanor et al., 2009). 
Les plantes médicinales et aromatiques jouent un rôle important dans la lute naturelle contre les champignons, c'est pour cela plusieurs essais ont été réalisés pour étudier l'effet de plante ou parties de plante sur la croissance des champignons ou la production des mycotoxines (Kwazou et al., 2009).

Dans le présent travail, l'activité antifongique de trois extraits aqueux de plante retenue à partir d'une enquête ethnobotanique réalisée dans la région du Moyen Atlas central, a été évalués par l'analyse de leur effet sur la croissance des champignons.

\section{Matériel Et Méthodes}

\section{Espèces végétales étudiées}

Sur la base d'une enquête ethnobotanique réalisée dans la région du moyen Atlas marocain, et en se référant aux données de la littérature et aux résultats du dosage des polyphénols, nous avons retenu trois espèces de plantes pour évaluer leur potentiel antifongique, Urginea maritima (L.) Baker famille des Liliacées, Chenopodium ambrosoides L. famille des Chenopodiacées et Calendula sp famille des Asteracées. En effet, ces espèces sont souvent utilisées localement dans le traitement de diverses affections dont celles de la peau causées par certains champignons.

Espèce 1 : Calendula officinalis $L$

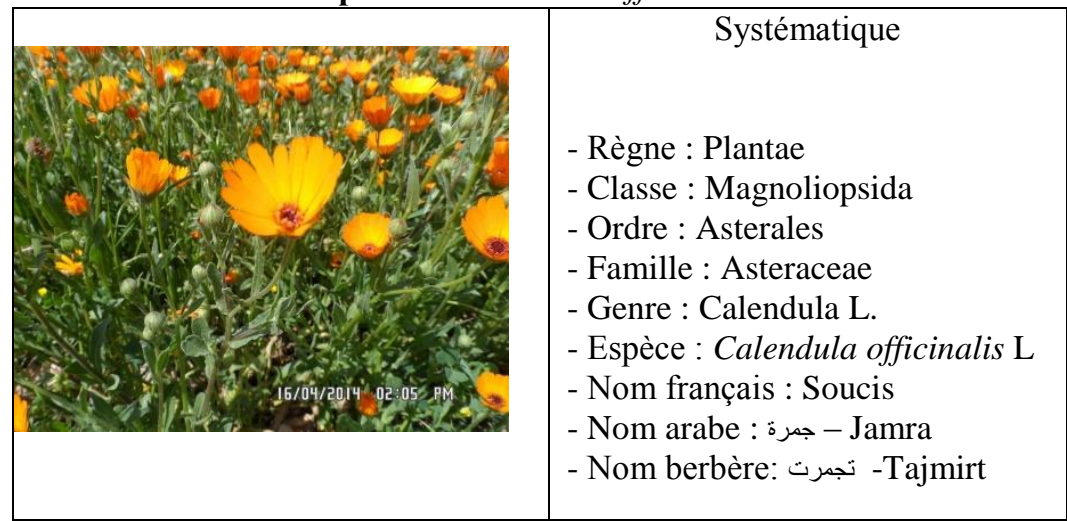

Description botanique : Herbe annuelle ou bisannuelles ou vivace. Feuille alterne, spatulé, obovées, oblancéolées, oblongues, lancéolées ou linéaires, uninervées, sommet obtus a acuminé : base atténuée, tronquée ou articulée; marge entières a plus ou moins sinuées-dentées, rarement pannatifides. Capitules solitaires, radiés, hétérogames ; involucre a branche 1(-n) sériées. Feuille périphériques ligulées, jaunes ou orange, femelles, fertiles, disposées en 1(-n) rangées. Fleurons centraux males, jaunes, oranges, marron ou violet pourpre; corone tubulaire, 5-lobée, non 
sacciforme. Akène hétéromorphes : les externes en général rostrées, droit à fortement courbés, avec ou sans épines dorsales, parfois 2-3 ailés; les médians soit 2-3 ailés, ailes latérales dentées ou entières, soit naviculaires, avec ou sans aile ventrale ; les internes vermiculaires, falciformes ou quasiannulaires aptères ou avec 2 ailes latérales étroites, tuberculés-rugueux sur le dos (Fennane et al., 2014).

Usage traditionnels: Le souci était déjà utilisé en cuisine, en médecine et en cosmétique par les civilisations indiennes, arabes et grecques de l'antiquité. Les fleurs et les racines de cette plante sont utilisées pour Traiter les inflammations de la peau, eczéma et des muqueuses de la bouche et de la gorge, les plaies et les ulcères variqueux, antiseptique, antifongique, antiviral, cholérétique et emménagogue (Goetz, 2009). Cette plante est utilisée selon notre enquête du terrain pour traiter les pathologies dermiques avec un facteur de fidélité égale à $18.96 \%$, aussi utilisée pour traiter les infections bactériennes de la sphère oto-rhino-laryngologie (Daoudi et al., 2015).

De même, Bammou et al (2015) ont rapporté l'usage de cette espèce pour traiter les affections du tube digestif, dermatologiques, et celles de système respiratoire (Bammou et al., 2015).

Le souci est capable d'offrir une cardioprotection en stimulant la pression du ventricule gauche, le flux aortique, en réduisant la taille d'un éventuel infarctus myocardique, et l'apoptose des cardiomyocytes. Il modifie le signal létal par ischémie en un signal de survie en modifiant la voie de l'oxydation et de l'inflammation (Ghédira \& Goetz, 2016).

Des travaux scientifiques ont été effectués pour confirmer ou infirmer certains usages traditionnels de cette plante comme les propriétés antiinflammatoires, antiseptiques, antibactériennes et emménagogues (Hmamouchi, 1999).

Toxicité : La toxicité orale subchronique d'un extrait de Calendula a été évaluée chez des rats Wistar mâles et femelles. Plusieurs des éléments de sang ont été affectés de manière significative chez les mâles et les femelles après 90 jours : l'hémoglobine, les leucocytes et les érythrocytes, le temps de coagulation du sang. L'examen histopathologique des tissus a montré de légères anomalies dans le parenchyme hépatique (Ghédira \& Goetz, 2016).

Chimie de la plante: L'huile essentielle $(0,12 \%$ dans les pétales et $0,4 \%$ dans les inflorescences), Flavonoides (0,88\% dans les pétales, 0,33\% dans les inflorescences), Saponines triterpèniques, Alcools triterpéniques, libres et estérifiés (monols, diols, triols), Polysaccharides (15\%) solubles 
dans l'eau, Caroténoides et xanthophylles $(0,017$ à $0,078 \%)$ (Ghédira \& Goetz, 2016).

Espèce 2 : Urginea maritima (L.) Baker

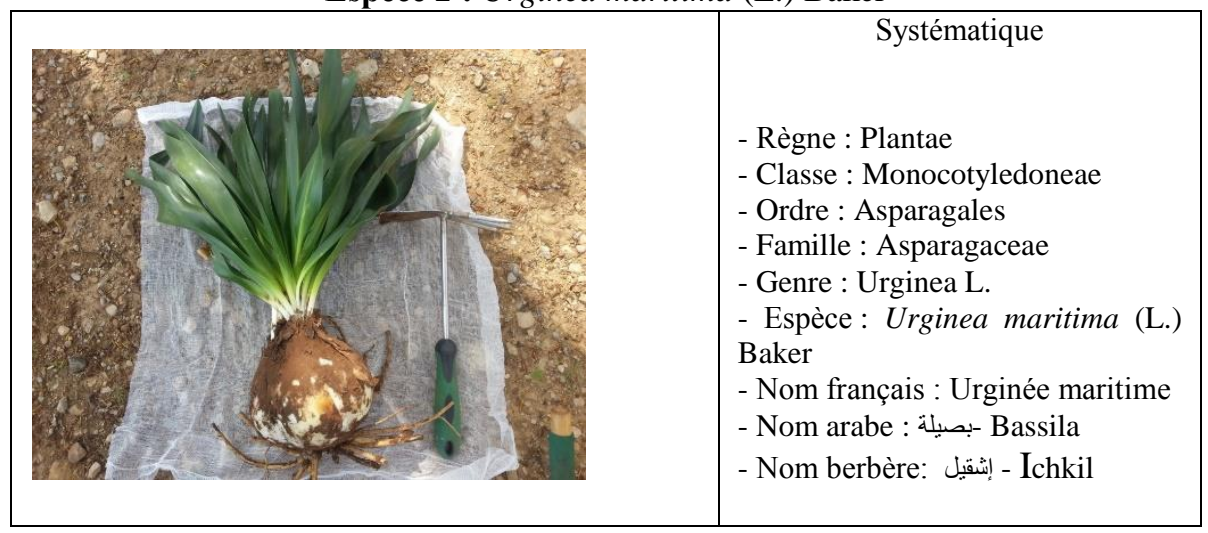

Description botanique: Feuille glabre, à marge lisse. Périanthe étoilé ; tépale brièvement soudés à la base, long 8-12 mm, blanc, à une seule nervure pourpre ou verte. Bractée plus courtes que les pédicelles. Filets blanc; anthères basifixes. Graines noires, brillante. Géophyte a bulbe. Clairières, milieux rudéraux, rochers, rocailles, plaines, basses et moyens montagne- Aride, semi-aride doux, semi-aride froid, subhumide, humide. Répartition : Se trouve dans tous le Maroc (Fennane et al., 2014).

Utilisation médicinale : Selon notre enquête réalisée dans la région du Moyen Atlas central, cette plante est utilisée pour traiter les problèmes dermatologiques avec un indice de fidélité de $17.50 \%$, en plus cette pathologie, elle est utilisée sous forme de goutte sur les yeux pour traiter les douleurs de l'oreille et ceci après broyage et filtration du bulbe (El Hafian et al., 2014). Aussi elle utilisée pour traiter les pathologies digestives (Mehdioui \& Kahouadji, 2007), les problèmes cardiaques, la coqueluche (Hmamouchi, 1999), cette plante est recommandée pour traiter aussi l'hépatite (Bellakhdar, 1997; Benkhnigue et al., 2010). Les femmes de la compagne l'utilisent comme abortif par voie interne ou en fumigations vaginales. Selon (Deepak et al., 2003), un extrait des bulbes de Urginea a présenté une activité antifongique puissant et à large spectre contre de nombreux champignons pathogènes.

Toxicité : Cette plante est toxique pour l'homme et l'animal, qui se manifeste par des vertiges, des nausées, des vomissements, des diarrhées, de l'hypertension, des douleurs dans le ventre et dans les jambes. Dans des cas 
graves survient un coma puis la mort du a un arrêt cardiaque (Bellakhdar, 1997; Hmamouchi, 1999).

Chimie de la plante : Des hétérosides cor diatomiques stériques sont les principaux principes actifs de cette plante (Bellakhdar, 1997; Hmamouchi, 1999).

Espèce 3 : Chenopodium ambrosioides L.

\begin{tabular}{|l|l|}
\hline & \multicolumn{1}{|c|}{ Systématique } \\
& - Règne : Plantae \\
- Classe : Magnoliopsida \\
- Ordre : Caryophyllales \\
- Famille: Chenopodiaceae \\
- Genre:Chenopodium L. \\
- Espèce: Chenopodium ambrosioides \\
L.
\end{tabular}

Description botanique : Plante annuelle ou vivaces, herbacées ou suffrutescentes, glabre, farineuses ou poilues-glanduleuses. Tige plus ou moins sillonnées. Feuille à dents espacées sur les marges, nervures peu saillantes en dessous. Inflorescence en epi ou en panicule de glomérules axillaires. Fruit akène à péricarpe scarieux. Graine horizontale ou verticale, lenticulaire, lisse ou ponctuée noiratre et luisante. Plante thérophyte (printemps, été et automne), naturalisée dans les milieux inclûtes frais, fossés, haies et alluvions sablonneuses. Bioclimat : aride semi-aride doux, subhumide.

Répartition: Se trouve dans le Maroc saharien, haut atlas, Maroc atlantique moyen, Maroc atlantique nord, plateau du Maroc oriental, le littorale de la méditerrané et le rif (Fennane \& Ibn-Tattou, 1999).

Utilisation traditionnelles : Selon (Bellakhdar, 1997; Daoudi et al., 2015; Lahsissene et al., 2009), cette espèce est parmi les plantes les plus sollicités de la région du Moyen Atlas central pour ces vertus médicinales. En effet, elle utilisés pour soulager les problèmes rhumatologiques, respiratoires et gastriques. De même, cette plante est utilisée pour traiter les problèmes dermatologiques causés par les champignons, et pour traiter les maladies de l'appareil circulatoire (Hseini et al., 2007). Egalement, les graines de cette plante sont utilisées comme vermifuge, contre les abcès buccaux, les ulcérations et des plaies purulentes, par application locale (Bellakhdar, 1997). L'huile essentielle de cette plante présente une 
importante activité antalgique de type non morphinique. (Javier et al., 1991; Ongoka et al., 2003) ont mentionnés que Chenopodium ambrosioides L. a une activité antifongique remarquable.

Toxicité : Cette plante est assez toxique, surtout chez l'enfant, cette intoxication se manifeste par des perturbations du système nerveux centrale, des nausées et des vomissements sont également signalé suite à la prise de cette plante (Benkhnigue et al., 2010). Parfois on observe une inflammation des intestins, une hypotension et des hémorragies importantes au niveau des méninges et des intestins (Bellakhdar, 1997).

Chimie de la plante: L'étude chimique de la plante a révèle la présence de nombreux constituants chimiques notamment les alcaloïdes, les quinones, les tanins et les terpènes ainsi que l'absence des anthocyanes et des composés poly phénoliques (Ongoka et al., 2003).

\section{Préparation des extraits aqueux}

L'extrait aqueux est préparé via les trois modes d'extraction traditionnelle à savoir : la décoction, l'infusion et la macération.

Extraction par infusion : $10 \mathrm{~g}$ de poudre est ajouté à $100 \mathrm{ml}$ d'eau bouillon et mise en incubation pendant $15 \mathrm{~min}$, le mélange obtenu est filtré sur papier filtre, le filtrat est mis dans l'étuve pour l'évaporation.

Extraction par décoction : $100 \mathrm{ml}$ d'eau est ajouté à $10 \mathrm{~g}$ de poudre et mis jusqu'à ébullition pendant 15 min puis filtré sur papier filtre avant d'être séché dans l'étuve à $50^{\circ} \mathrm{C}$.

Extraction par macération : $10 \mathrm{~g}$ de poudre et $100 \mathrm{ml}$ d'eau sont mis sous agitation mécanique pendant $48 \mathrm{~h}$ à température ambiante, le mélange obtenu est filtré et le filtrat est mis dans l'étuve pour l'évaporation d'eau.

\section{Quantification des principes actifs}

\section{Dosage des polyphénols}

Les polyphénols sont mesurés par la méthode Folin-Ciocalteu, un mélange d'extrait aqueux et du réactif du Folin est mis en incubation pendant 4 min avant d'ajouter $0,8 \mathrm{~mL}$ de carbonate de sodium et l'absorbance est mesurée au $\lambda_{\max }=765$ nanomètres. Les résultats sont exprimés en $\mathrm{mg}$ équivalent d'acide gallique par g d'extrait (Ghedadba et al., 2015).

\section{Matériel fongique}

Les souches fongiques utilisées, ont été procuré du laboratoire de biotechnologie végétale à la Faculté des Sciences et Techniques d'Errachidia, à savoir : Fusarium oxysporum f. sp. albedinis, Alternaria sp, Penicillium expansum, Aspergillus brasiliensis et en fin Rhizopus stolonifer. 


\section{Préparation de l'inoculum}

On prélève avec un emporte-pièce des disques mycéliens de $6 \mathrm{~mm}$ de diamètre à partir de la périphérie d'un tapis mycélien provenant d'une culture de $7 \mathrm{j}$.

\section{Préparation des boites de pétri}

La méthode utilisée consiste à incorporer aseptiquement dans 3 erlens contenant $100 \mathrm{ml}$ de milieu PDA stérile, des volumes de $8 \mathrm{ml}, 4 \mathrm{ml}$ et $2 \mathrm{ml}$ de la solution mère, l'ensemble est rapidement homogénéisé. L'incorporation des différents volumes au milieu PDA a conduit à l'obtention des concentrations finales suivantes $10,71 \mathrm{mg} / \mathrm{ml}, 5,66 \mathrm{mg} / \mathrm{ml}$ et $1,96 \mathrm{mg} / \mathrm{ml}$.

$\mathrm{Au}$ centre de chaque boîte content le mélange de milieu PDA et de la concentration de l'extrait à tester, on dépose le disque du champignon, une série de boites de pétri a été préparée.

L'incubation se fait à l'obscurité à une température de $25 \pm 2{ }^{\circ} \mathrm{C}$ pendant 3 jours pour Rhizopus stolonifer et 6 jours pour Alternaria sp., et Penicillium expansum, Fusarium oxysporum et Aspergillus brasiliensis. Les durées d'incubations ont été fixées après une étude préliminaire sur les vitesses de croissance des moisissures sélectionnées.

Pour chaque espèce fongique et chaque concentration, trois répétitions ont été réalisées et trois boîtes sont utilisées par essai. La croissance mycélienne a été suivie en mesurant la moyenne de deux diamètres perpendiculaires passant par le centre de chaque boite. La fongitoxicité, exprimée en terme de pourcentage d'inhibition de la croissance de mycélium (IP \%), a été calculée selon la formule de (Rapilly, 1968).

$$
I P \%=\left(1-\left(\frac{\mathrm{Dc}}{\mathrm{Dt}}\right)\right) \times 100
$$

PI : Pourcentage d'inhibition de la croissance mycélienne ;

$D_{C}$ : Diamètre des disques mycéliens pour une concentration donnée ;

$\mathrm{D}_{\mathrm{T}}$ : Diamètre des disques mycéliens témoins.

\section{Resultats Et Discussion Dosage des polyphénols}

Les polyphénols sont parmi les principes actifs de plante qui sont distribués dans le règne végétal et sont consommés sous différente forme. Ils sont capables de modifier le comportement de plusieurs systèmes cellulaires, 
ils sont reconnus comme des molécules antibactériennes, antifongiques, antiinflammatoires, antioxydants, et même antitumorales (Kouakou et al., 2006). Le dosage des polyphénols des différents extraits des plantes étudiées a révélé que le décocté de Chenopodium ambrosioides L. présente la quantité la plus importante. En effet, elle présente une quantité (Qt) équivalente d'acide gallique $(\mathrm{Ag})$ en $\mathrm{mg}$ par $1 \mathrm{~g}$ d'extrait équivalente à 82,42 Qt eq $\mathrm{Ag}$ $\mathrm{mg} / \mathrm{g}$ d'extrait. Suivie de l'infusé (69,25 Qt eq Ag mg/lg d'extrait) et en fin de macérât (49,45 Qt eq Ag mg/1g d'extrait) (Figure 1).

Les résultats obtenus pour Calendula officinalis L. sont rapporté dans la figure 2, l'infusé de Calendula officinalis L. a présenté la quantité la plus importante en polyphénol par rapport aux autres extraits, 68,69 Qt eq Ag $\mathrm{mg} / \mathrm{g}$ d'extrait. Alors que, la quantité la plus élevée en polyphénol est observée pour le macérât de Urginea maritima (L.) Baker (60,90 Qt eq Ag $\mathrm{mg} / 1 \mathrm{~g}$ d'extrait) (Figure 3).

En général, les trois espèces étudiées renferment des concentrations non négligeables en polyphénols, susceptibles d'exprimer les activités antifongiques recherchées. La présence des polyphénols dans les différents extraits des plantes étudiées est également rapportée par plusieurs auteurs (Javaid \& Amin, 2009; Mammadov et al., 2010; Pop et al., 2009).

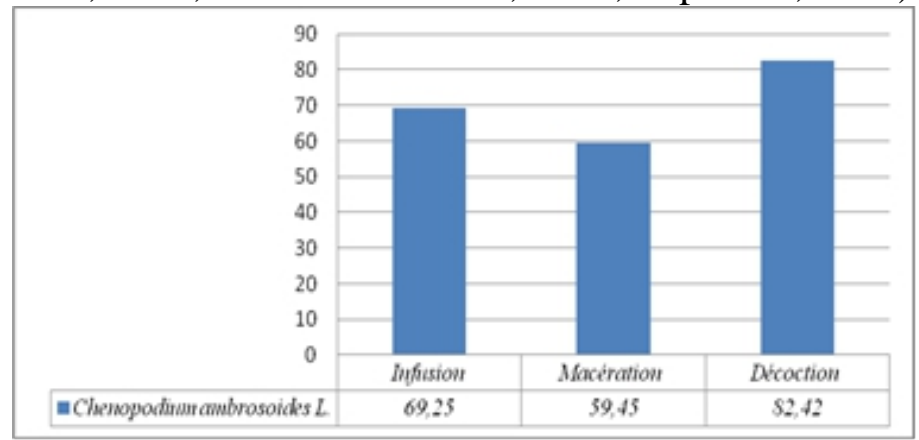

Figure1 : Dosage des polyphénols Chenopodium ambrosioides L.

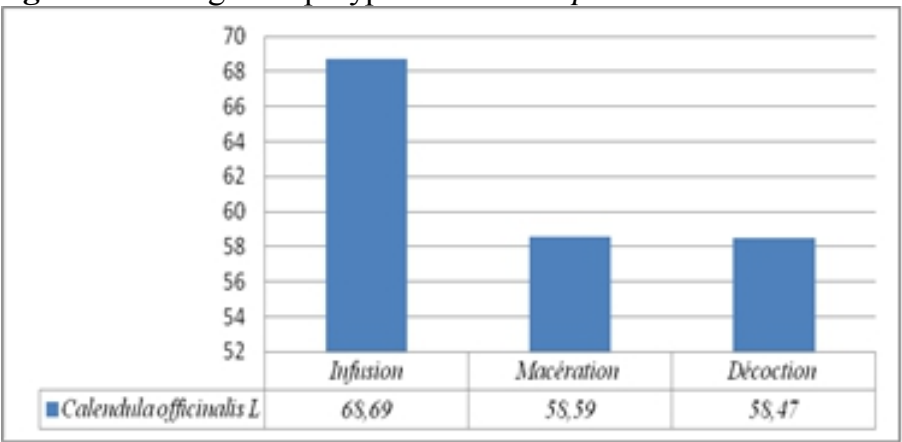

Figure 2 : Dosage des polyphénols Calendula officinalis L. 
e Crginea maritima $($ L. Baker

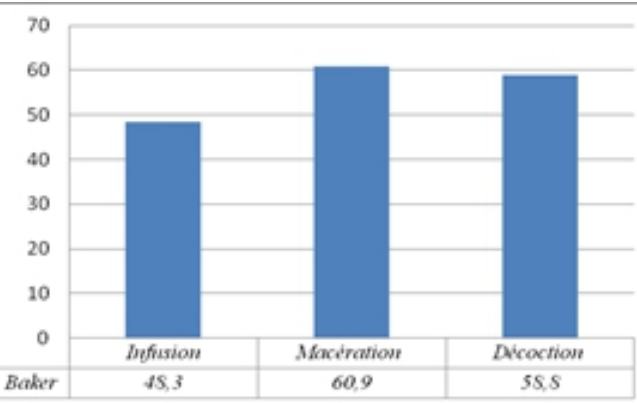

Figure 3 : Dosage des polyphénols Urginea maritima (L.) Baker

\section{Activité antifongique}

Cinq souches ont été sélectionnées pour servir à déceler l'activité antifongique des extraits aqueux des espèces étudiées à savoir : Fusarium oxysporum, Alternaria sp, Penicillium expansum, Aspergillus brasiliensis et Rhizopus stolonifer.

Les résultats de l'activité antifongique des extraits aqueux d'Urginea maritima (L.) et Chenopodium ambrosioides L, testés séparément in vitro sur les souches fongiques sélectionnées sont représentés dans les tableaux 1 et 2. L'extrait aqueux de Calendula officinalis L. n'a exprimé aucune activité.

Ces tableaux enregistrent les pourcentages d'inhibition de la croissance mycélienne notés pour chaque concentration d'extrait testée (C1, C2 et C3).

Le résultat de l'extrait aqueux d'Urginea maritima (L.) testé contre les différentes espèces de champignons sur milieu PDA, indique une activité antifongique positive sur Aspergillus brasiliensis, Fusarium oxysporum, et Alternaria $s p$, avec un PI égale respectivement, 43,33\%, 19,25\% et $10 \%$, pour une concentration de $\mathrm{C} 1=10,71 \mathrm{mg} / \mathrm{ml}$. Alors que le pourcentage d'inhibition de la croissance mycélienne est apparu nul pour Penicillium expansum et Rhizopus stolonifer sur toute la gamme de concentration utilisée.

Les effets antifongiques d'extrait aqueux d'Urginea maritima (L.) peuvent être attribués aux polyphénols de cette plante, mais certaines études ont démontré que l'activité antifongique d'Urginea maritima (L.) est liée à une glycoprotéine localisée dans la paroi cellulaire de l'ampoule, qui inhibe complètement la germination des spores et la croissance des hyphes de Fusarium oxysporum (Deepak et al., 2003).

Concernant les résultats de l'extrait aqueux de Chenopodium ambrosioides L, cette plante est active, avec une concentration de 10,71 $\mathrm{mg} / \mathrm{ml}$, sur toutes les souches fongiques testées, et les pourcentages d'inhibition de la croissance mycélienne enregistrés sur Aspergillus brasiliensis, Alternaria sp, Fusarium oxysporum, Penicillium expansum et Rhizopus stolonifer, sont respectivement, 41,11\%, 40,37\%, 27,77\%, 10,74 $\%$ et $10 \%$. Cette plante est aussi active avec une concentration de 5,66 
mg/ml, sur Aspergillus brasiliensis, Alternaria sp et Fusarium oxysporum, avec un pourcentage d'inhibition de la croissance mycélienne égale respectivement à $20 \%, 18.88 \%$ et 9,25\%. Ces résultats concordent avec ceux rapportés par (Shah, 2014), qui ont confirmé l'activité antifongique des extraits aqueux de Chenopodium ambrosioides L, sur les souches du genre Aspergillus et Rhizopus.

Aucune concentration de l'extrait aqueux de Calendula officinalis L. testé sur les souches fongiques n'a montrée aucune activité.

Le mécanisme d'action des extraits des plantes a été évalué par plusieurs études. Certains chercheurs attribuent cette fonction aux composés phénoliques qui sont douées d'une activité antifongique. Ces composés peuvent altérer la structure de la membrane cellulaire, on inhibant la croissance mycélienne (Ghani et al., 2008; Yan et al., 2008).

Néanmoins, malgré la présence de quantité suffisante de polyphénols, certaines plantes sont dénuées d'activité biologique, que ce soit antibactérienne, antifongiques ou insecticides, c'est le cas de Calendula officinalis L. qui n'a pas présenté d'activité antifongique vis-à-vis des souches fongiques testées, malgré une quantité remarquable de polyphénols égale à 68,96 Qt eq Ag mg/g d'extrait.

La teneur en principes actifs, peut différer selon les variations de climat, type de sol, les conditions de récolte et autres facteurs qui peuvent expliquer l'effet thérapeutique d'une plante. L'ensemble de ces substances actives appelé aussi le totum de plante, fonctionne en synergie. Il est plus efficace que le principe actif isolé, La plante dans son totum présente des potentialités d'action très variées (Amar et al., 2010).

Tableau 1 : Pourcentages d'inhibition de la croissance mycélienne d'Urginea maritima (L.)

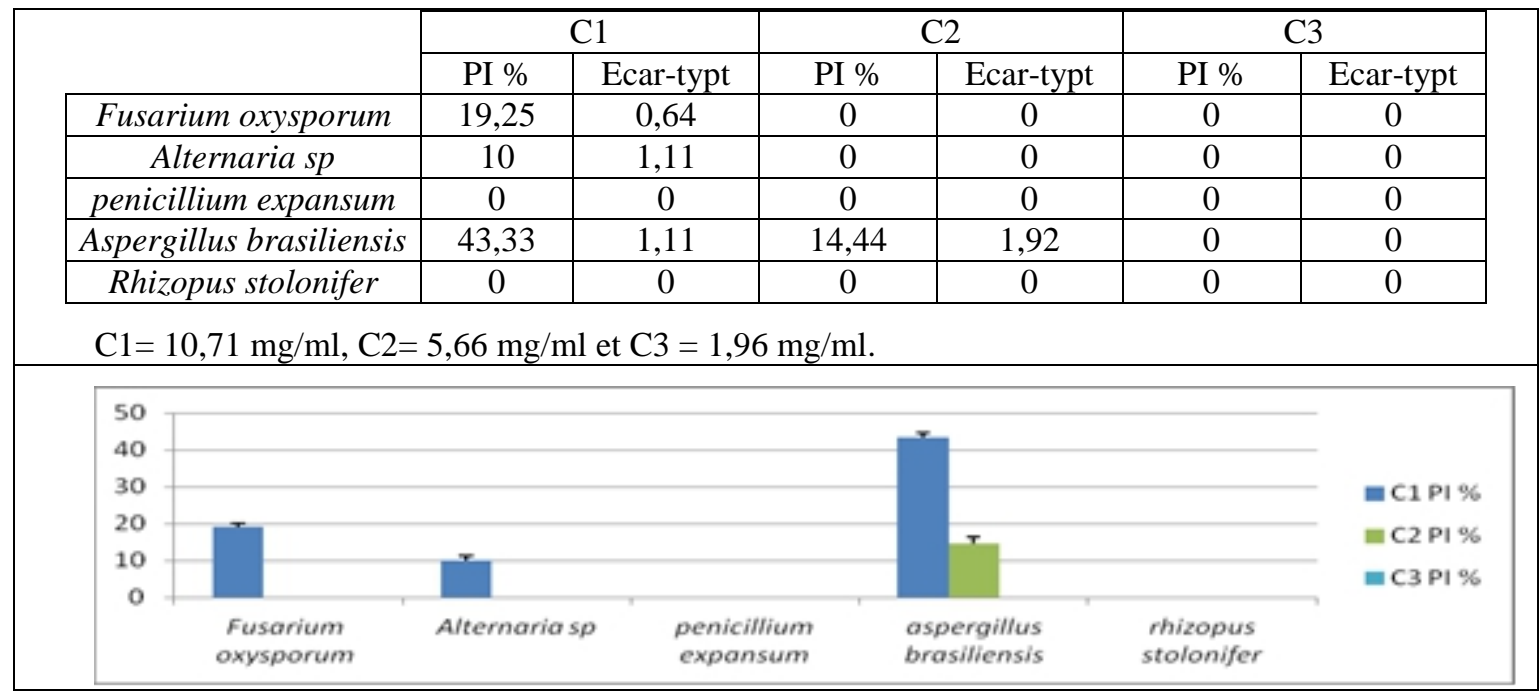


Tableau 2 : Pourcentages d'inhibition de la croissance mycélienne de Chenopodium ambrosoides L.

\begin{tabular}{|c|c|c|c|c|c|c|}
\hline \multirow{2}{*}{} & \multicolumn{2}{|c|}{ C1 } & \multicolumn{2}{c|}{ C2 } & \multicolumn{2}{c|}{ C3 } \\
\cline { 2 - 7 } & PI \% & Ecar-typt & PI \% & Ecar-typt & PI \% & Ecar-typt \\
\hline Fusarium oxysporum & & & & & & \\
& 27,77 & 1,11 & 9,25 & 1,69 & 0 & 0 \\
\hline Alternaria sp & 40,37 & 1,69 & 18,88 & 1,11 & 0 & 0 \\
\hline Penicillium expansum & 10,74 & 0,64 & 0 & 0 & 0 & 0 \\
\hline Aspergillus brasiliensis & 41,11 & 1,11 & 20 & 1,11 & 0 & 0 \\
\hline Rhizopus stolonifer & 10 & 1,11 & 0 & 0 & 0 & 0 \\
\hline
\end{tabular}

$\mathrm{C} 1=10,71 \mathrm{mg} / \mathrm{ml}, \mathrm{C} 2=5,66 \mathrm{mg} / \mathrm{ml}$ et $\mathrm{C} 3=1,96 \mathrm{mg} / \mathrm{ml}$.

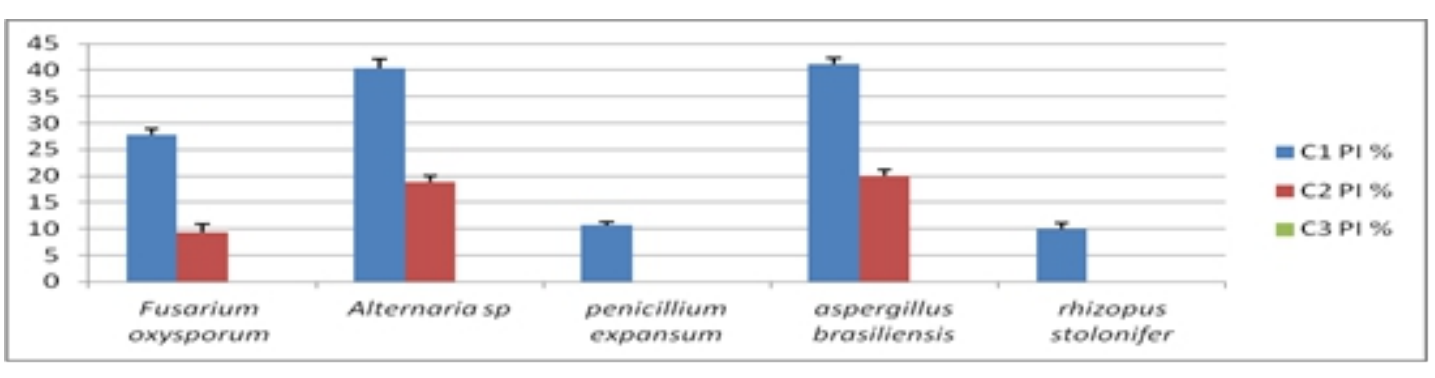

\section{Conclusion}

L'étude de l'activité antifongique des extraits aqueux des trois espèces de plantes étudiées de la région du Moyen Atlas Central, a révélé que l'extrait aqueux d'Urginea maritima (L.) est actifs vis-à-vis d'Aspergillus brasiliensis, Fusarium oxysporum, et Alternaria sp, avec un PI égale respectivement, $43,33 \%, 19,25 \%$ et $10 \%$, pour une concentration de $\mathrm{C} 1=10,71 \mathrm{mg} / \mathrm{ml}$. Pour cette même concentration, l'extrait aqueux de Chenopodium ambrosioides L, exerce une activité antifongique vis-à-vis d'Aspergillus brasiliensis, Alternaria sp, Fusarium oxysporum, Penicillium expansum et Rhizopus stolonifer, avec un PI respectivement de, 41,11\%, $40,37 \%, 27,77 \%, 10,74 \%$ et $10 \%$. Cette plante est aussi active avec une concentration de 5,66 mg/ml, contre Aspergillus brasiliensis, Alternaria sp et Fusarium oxysporum, avec un pourcentage d'inhibition de la croissance mycélienne égale respectivement à $20 \%, 18.88 \%$ et 9,25\%. Les deux extraits aqueux possèdent une remarquable activité antifongique liée, peutêtre, à leur richesse en composés bioactifs largement répandus dans les plantes médicinales.

\section{References:}

1. Amar, A., Harrache, D., Atmani, F., Bassou, G., \& Grillon, F. (2010). Effet de Parietaria officinalis sur la cristallisation de l'oxalate de calcium, dans l'urine. Phytothérapie, 8(6), 342-347. 
2. Badji, B., Riba, A., Mathieu, F., Lebrihi, A., \& Sabaou, N. (2005). Activité antifongique d'une souche d'Actinomadura d'origine saharienne sur divers champignons pathogènes et toxinogènes. Journal de Mycologie Médicale/Journal of Medical Mycology, 15(4), 211-219.

3. Bammou, M., Daoudi, A., Sellam, K., El Rhaffari, L., Ibijbijen, J., \& Nassiri, L. (2015). Ethnobotanical Survey of Asteraceae Family used in Meknes-Tafilalet Region (Morocco). International Journal of Innovation and Applied Studies, 13(4), 789-815.

4. Bellakhdar, J. (1997). La pharmacopée marocaine traditionnelle. Médecine arabe ancienne et savoirs populaires. . Editions Le Fennec, Casablanca. 764 pp: Ibis Press.

5. Benkhnigue, O., Zidane, L., Fadli, M., Elyacoubi, H., Rochdi, A., \& Douira, A. (2010). Etude ethnobotanique des plantes médicinales dans la région de Mechraâ Bel Ksiri (Région du Gharb du Maroc). Acta Botanica Barcinonensia, 53, 191-216.

6. Daoudi, A., Bachiri, L., Bammou, M., Ibijbijen, J., \& Nassiri, L. (2015). ETUDE ETHNOBOTANIQUE AU MOYEN ATLAS CENTRAL. European Scientific Journal, 11(24).

7. Deepak, A., Thippeswamy, G., Shivakameshwari, M., \& Salimath, B. P. (2003). Isolation and characterization of a $29-\mathrm{kDa}$ glycoprotein with antifungal activity from bulbs of Urginea indica. Biochemical and Biophysical research communications, 311(3), 735-742.

8. El Hafian, M., Benlandini, N., Elyacoubi, H., Zidane, L., \& Rochdi, A. (2014). Étude floristique et ethnobotanique des plantes médicinales utilisées au niveau de la préfecture d'Agadir-IdaOutanane (Maroc). Journal of Applied Biosciences, 81(1), 71987213.

9. Fennane, M., \& Ibn-Tattou, M. (1999). Flore pratique du Maroc. Vol. 1. Pteridophyta, Gymnospermae, Angiospermae (LauraceaeNeuradaceae): Inst. Scientifique.

10. Fennane, M., Ibn-Tattou, M., \& El oualidi, j. (2014). Flore pratique du Maroc. Vol. 3. Dicotyledones, Monocotyledones: Inst. Scientifique.

11. Ghani, S. B. A., Weaver, L., Zidan, Z. H., Ali, H. M., Keevil, C. W., \& Brown, R. C. (2008). Microwave-assisted synthesis and antimicrobial activities of flavonoid derivatives. Bioorganic \& medicinal chemistry letters, 18(2), 518-522.

12. Ghedadba, N., Hambaba, L., Ayachi, A., Aberkane, M., Bousselsela, H., \& Oueld-Mokhtar, S. (2015). Polyphénols totaux, activités antioxydante et antimicrobienne des extraits des feuilles de Marrubium deserti de Noé. Phytothérapie, 13(2), 118-129. 
13. Ghédira, K., \& Goetz, P. (2016). Calendula officinalis L.(Asteraceae): souci. Phytothérapie, 14(1), 62-67.

14. Goetz, P. (2009). Phytothérapie de l'eczéma. Phytothérapie, 7(4), 211-214.

15. Hmamouchi, M. (1999). Les plantes médicinales et aromatiques marocaines. Rabat. 389pp: Editions FEDALA.

16. Hseini, S., Kahouadji, A., Lahssissene, H., \& Tijane, M. (2007). Analyses floristique et ethnobotanique des plantes vasculaires médicinales utilisées dans la région de Rabat (Maroc occidental). Lazaroa, 28, 93-100.

17. Javaid, A., \& Amin, M. (2009). Antifungal activity of methanol and n-hexane extracts of three Chenopodium species against Macrophomina phaseolina. Natural product research, 23(12), 11201127.

18. Javier, S. H. F., Roberto, G. E., \& Bertha, T. B. (1991). Efecto de la incorporacion de residuos secos de las plantas gobernadora (Larrea tridentata L.) y epazote (Chenopodium ambrosioides L.) en suelos infestados con Phythium aphanidermatum y Rhizoctonia solani, en la germinacion y crecimiento de plantas de frijol. Revista Mexicana de Fitopatologia.

19. Kouakou, A., N Guessan, J., Kra, A., \& Guédé-Guina, F. (2006). Activité antifongique et screening phytochimique de THOS (extrait aqueux de Thonningia sanguinea). JOURNAL-SOCIETE OUEST AFRICAINE DE CHIMIE, 22, 21.

20. Kwazou, N., Dongmo, P. M. J., Ngoune, L. T., Sameza, M., Dongmo, B., Zollo, P. H. A., \& Menut, C. (2009). Propriétés antifongiques des huiles essentielles de quelques plantes du genre Aframomum du Cameroun contre Aspergillus flavus. Cameroon Journal of Experimental Biology, 5(1).

21. Lahsissene, H., Kahouadji, A., \& Hseini, S. (2009). Catalogue des plantes medicinales utilisees dans la region de Zaër (Maroc Occidental). Lejeunia, Revue de Botanique(0457-4184), 30.

22. Mammadov, R., Makasç1-Afacan, A., Uysal-Demir, D., \& Görk, Ç. (2010). Determination of antioxidant activities of different Urginea maritima (L.) Baker plant extracts. Iranian Journal of Chemistry and Chemical Engineering (IJCCE), 29(3), 47-53.

23. Mehdioui, R., \& Kahouadji, A. (2007). Etude ethnobotanique auprès de la population riveraine de la forêt d'Amsittène: cas de la Commune d'Imi n'Tlit (Province d'Essaouira). Bulletin de l'Institut scientifique, Rabat, section Sciences de la vie, 29, 11-20.

24. Ongoka, P., Abena, A., Okémi-Andissa, N., Ouabonzii, A., \& Ekouya, A. (2003). Etude ethnobotanique, chimique et 
pharmacologique de deux plantes à activité anthelminthique et antalgique: Alchornea cordifolia et Chenopodium ambrosioides. Phytothérapie, 1(4), 106-110.

25. Pop, G., Alexa, E., \& Militaru, A. V. (2009). Antioxidant polyphenol and flavone contents in correlation with cultivation technology for Calendula officinalis L. Ann Oradea Univ Biol Fasc, 16, 102-104.

26. Rapilly, F. (1968). Les techniques de mycologie en pathologie végétale. 101.

27. Sauphanor, B., Simon, S., Boisneau, C., Capowiez, Y., Rieux, R., Bouvier, J.-C., . . . Toubon, J.-F. (2009). Protection phytosanitaire et biodiversité en agriculture biologique. Le cas des vergers de pommiers. Innovations agronomiques, 4, 217-228.

28. Shah, H. (2014). Antibacterial and antifungal activities of the crude extracts from the stem of Chenopodium ambrosioides Linn., an indiginous medicinal plant. African Journal of Pharmacy and Pharmacology, 8(8), 231-234.

29. Yan, D., Jin, C., Xiao, X.-H., \& Dong, X.-P. (2008). Antimicrobial properties of berberines alkaloids in Coptis chinensis Franch by microcalorimetry. Journal of biochemical and biophysical methods, 70(6), 845-849. 